\title{
Improving in-row weed detection in multispectral stereoscopic images
}

\author{
A. Piron*, V. Leemans, F. Lebeau, M.-F. Destain \\ Gembloux Agricultural University, Unité de Mécanique et Construction, 2 Passage des Déportés, 5030 Gembloux, Belgium
}

\section{A R T I C L E I N F O}

\section{Article history:}

Received 4 February 2009

Received in revised form 29 May 2009

Accepted 5 July 2009

\section{Keywords:}

Weed detection

Stereovision

Multispectral

Row detection

\begin{abstract}
A B S T R A C T
Previous research has shown that plant height and spectral reflectance are relevant features to classify crop and weeds in organic carrots: classification based on height gave a classification accuracy (CA) of up to $83 \%$ while classification based on a combination of three multispectral bands gave a CA of $72 \%$.

The first goal of this study was to examine the simultaneous use of both height and multispectral parameters. It was found that classification rate was only slightly improved when using a feature set comprising both height and multispectral data (2\%).

The second goal of this study was to improve the detection method based on plant height by setting an automatic threshold between crop and weeds heights, in their early growth stage. This threshold was based on crop row determination and peak detection in plant height probability density function, corresponding to the homogeneous crop population. Using this method, the CA was $82 \%$ while the CA obtained with optimal plant height limits is only slightly higher at $86 \%$.
\end{abstract}

(c) 2009 Elsevier B.V. All rights reserved.

\section{Introduction}

In-row weed detection by machine vision in organic carrots poses particular difficulties that arise from the high competition between crop and weed, irregular and dense sowing pattern, various growth stages and similar color of crop and weed.

In the development of weed detection machine vision systems, several approaches have been tested with various degrees of success. Among the most common methods, the use of spectral reflectance as a discriminant feature is widespread. However, the results are highly dependent on the species studied and the measurement conditions, both in terms of selected spectral band and classification results, so extrapolation to new data is not possible. Furthermore, narrow width and/or large number of spectral bands are also sometimes used which makes them poorly adapted to real time acquisition.

Using a spectrograph (660-1060 $\mathrm{nm}$ with a $5 \mathrm{~nm}$ resolution), Borregaard et al. (2002) studied potted beet, potatoes and three weed species at an early growth stage under artificial lighting. The best classification rate (89\%) was obtained by using two wavelengths (694 and $970 \mathrm{~nm}$ ) to discriminate sugar beet and weeds. For potatoes and weeds, the best classification reached 94\% using 856 and $686 \mathrm{~nm}$ wavelengths. Feyaerts and van Gool (2001) developed a spectrograph (435-1000 nm) with a low spectral resolution $(35 \mathrm{~nm})$ and used it in field to discriminate beets from five weeds

\footnotetext{
* Corresponding author. Tel.: +32 0816221 63; fax: +32 081622167 .

E-mail address: Piron.a@fsagx.ac.be (A.Piron).
}

species. The selected wavelengths were 441, 446, 459, 883, 924 and $988 \mathrm{~nm}$ with a correct classification rate of up to $83 \%$. Wang et al. (2001) developed an optical weed detector based on phototransistors. To select appropriate wavelengths, spectrometric measurements were performed on three weed species, wheat and soil at two growth stages, under laboratory conditions. The selected wavelengths were 496, 546, 614, 676 and $752 \mathrm{~nm}$. Measurements on potted plants were performed under artificial lighting. The classification rate between the weeds considered as a single group and the crop reached $72 \%$ for sufficiently densely weed infested samples (0.02 plants $\left./ \mathrm{cm}^{2}\right)$. For lower infestation rates or single plants, the classification rate was below 50\%. Vrindts et al. (2002) compared the results from a laboratory study on leaves using a spectrometer (400-2000 $\mathrm{nm}$ spectral range, $10 \mathrm{~nm}$ resolution) to in field natural lighting spectrography ( $480-820 \mathrm{~nm}, 2.1 \mathrm{~nm}$ resolution) results. Two crop plants (beet and maize) and seven weed species were studied. A $100 \%$ classification rate was obtained when using $9 \mathrm{spec}-$ tral bands (555, 705, 725, 815, 915, 1455, 1475, 1535 and $1935 \mathrm{~nm})$ to discriminate corn from weeds and using 8 spectral bands to separate beets from weeds $(545,555,815,1085,1455,1665,1705,1875)$. For the spectrographic data, the classification rates are slightly lower (93\% with nine wavelengths for beet and 91\% using eight bands for corn). Lighting conditions strongly influenced the classification rates and the discrimination was only efficient with data taken under identical and constant lighting.

Using a camera with a filter wheel holding four filters (red, green, blue and infrared), Franz et al. (1991) took images of four weed species and soy leaves under controlled lighting. Five statistical features of the leaves images were used, the classification rate was up to $94 \%$ in optimal conditions (manually selected sam- 
ples, fixed position of the plants relative to the lighting system), but only $76 \%$ with an automatic method under varying lighting position. Overlapping leaves also lowered the classification results. Zhang and Chaisattapagon (1995) selected five filters to discriminate five species of weeds from soil and wheat at two growth stages, on potted plants located under artificial lighting. Piron et al. (2008a) used a combination of three wide band interference filters to detect weeds located within carrots rows and found a classification accuracy of only $72 \%$.

Another more recent image analysis family of methods was used for distinguishing plants. It is based on the measurement of plant height. Sanchez and Marchant (2000) described the possibility of detecting weeds by a fixed threshold on plant height on stereoscopic images of plants in laboratory conditions. Nielsen et al. (2004) studied the detection of weeds among tomato plants by analyzing stereoscopic images acquired in field by a trinocular camera. The distinction between crop and weeds was based on three different methods: simple per-pixel threshold on distance from camera to plant pixels, analysis of connected blobs height histograms and analysis of those same blobs after watershed segmentation. Authors acknowledged the negative effect of ground irregularity on classification. Other plant properties have also been studied on stereoscopic images. He et al. (2003) used stereoscopic images acquired by a binocular camera to evaluate average plant height and leaf area, among other parameters, on potted transplants. Andersen et al. (2005) studied the possibility of computing geometric plant properties such as plant height and leaf area on stereoscopic images acquired with a binocular camera, on isolated potted plants. They showed that those characteristics can be determined using stereovision but specified that the acquisition method needed to be validated for in-field conditions and for more complex plant structures. Piron et al. (2008b) showed that stereoscopic data of plants could be used to differentiate weeds from crop in organic carrots provided that actual plant height was computed and classification rules taking into account plant growth were used. A classification accuracy of $83 \%$ was reached.

However in all the previously mentioned studies the determination of the crop height range was dependent on manual training or operator input. This is not suitable for online application because plant growth and therefore height are dependent on many variables such as weather, soil condition...

Given the previous results showing the potential of both multispectral and stereoscopic methods, the first goal of this research was thus to assess the improvement gained by combining multispectral and stereoscopic data for weed detection among carrots. The second goal was to automate the discriminant parameter values determination for the height based weed detection, as those values are dependent on plant growth speed and weed species.

\section{Materials and methods}

\subsection{Multispectral and stereoscopic acquisition methods}

The multispectral acquisition device comprised a black and white camera (C-cam BCI 51.3 megapixels) coupled with a filter wheel holding 22 interference filters covering the visible and near infrared spectrum (400-1000 nm approximately) (Piron et al., 2008a).

The stereoscopic acquisition was based on the coded structured light technique. A bi-dimensional light pattern containing a known spatial information coding was projected using a video projector (Figs. 1 and 2). The deformed imaged patterns were acquired by the camera without filter. The depth information was obtained from the relationship between the captured and projected patterns (Piron et al., 2008b).

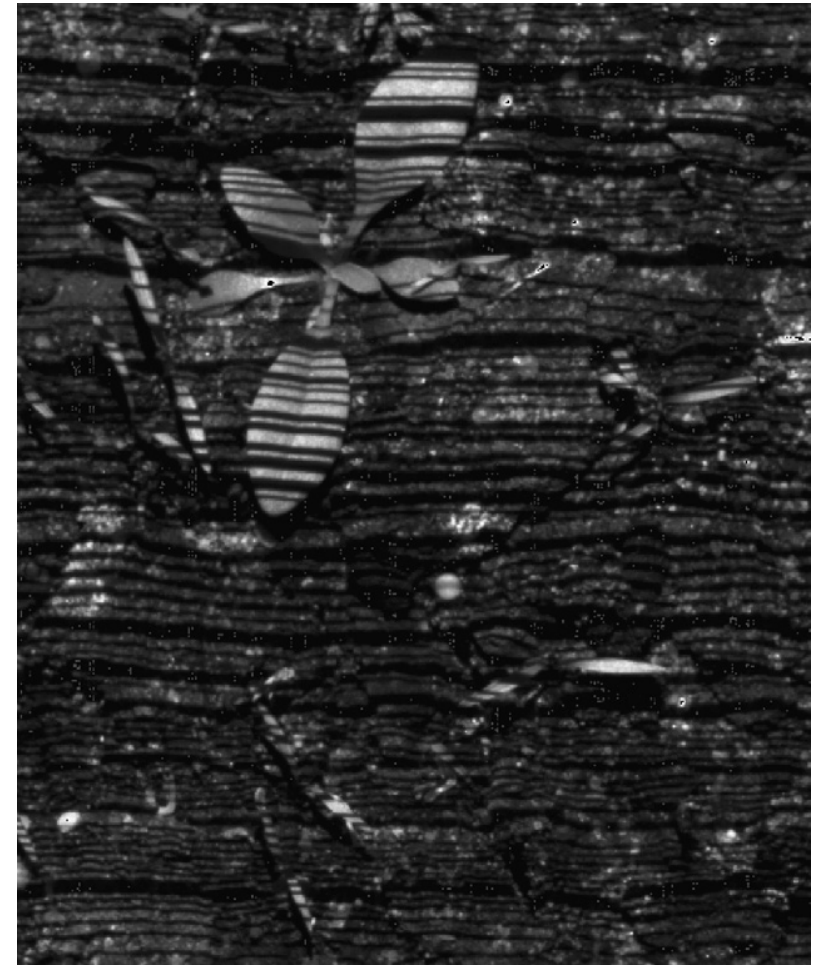

Fig. 1. Scene showing soil, a weed and carrot cotyledons with projected pattern (detail).

A mobile support frame was designed to allow acquisition of topdown images of the field scene (approximately 200 by $250 \mathrm{~mm}$ ). The scene was shielded from natural light and lit by a mixture of incandescent and fluorescent lights.

\subsection{Acquired data}

The study concerned two carrots varieties without distinction, Nerac F1 and Namur F1, sown on 27-04-06 in an experimental plot. Approximately 200 linear meters of rows were mechanically sown at a density of $10-15$ seeds per $100 \mathrm{~mm}$ long by $50 \mathrm{~mm}$ wide. Several species of weeds were naturally present in the field and others were manually introduced. The main species were the following at the time of data acquisition: Sonchus asper l, Chenopodium, Achenocloa Cirsium, M. perennis, Brassica and Matricaria maritime. Other species might have been present.

Images were acquired at an early growth stage of both carrots and weeds, 21, 28, 30, 37 and 39 days after sowing (this corresponded approximately to one week after crop emergence to 19 days later) which is the usual period for manual weeds removal. Indeed, early weed detection can increase yields and weed elimination becomes increasingly difficult with plant growth. In May, the soil was wet and dark, while it was dry and lighter in June (Table 1). A total of 28 stereoscopic multispectral images were acquired at random locations in the parcel over the measurement period. This

Table 1

Summary of acquired data.

\begin{tabular}{clll}
\hline $\begin{array}{c}\text { Date of data } \\
\text { acquisition }\end{array}$ & $\begin{array}{l}\text { Days after } \\
\text { sowing }\end{array}$ & $\begin{array}{l}\text { Soil surface } \\
\text { state }\end{array}$ & $\begin{array}{l}\text { Acquired multispectral } \\
\text { stereoscopic images }\end{array}$ \\
\hline $22-05-06$ & 21 & Wet & 4 \\
$29-05-06$ & 28 & Wet & 4 \\
$31-05-06$ & 30 & Wet & 9 \\
$07-06-06$ & 37 & Dry & 7 \\
$09-06-06$ & 39 & Dry & 4 \\
\hline
\end{tabular}



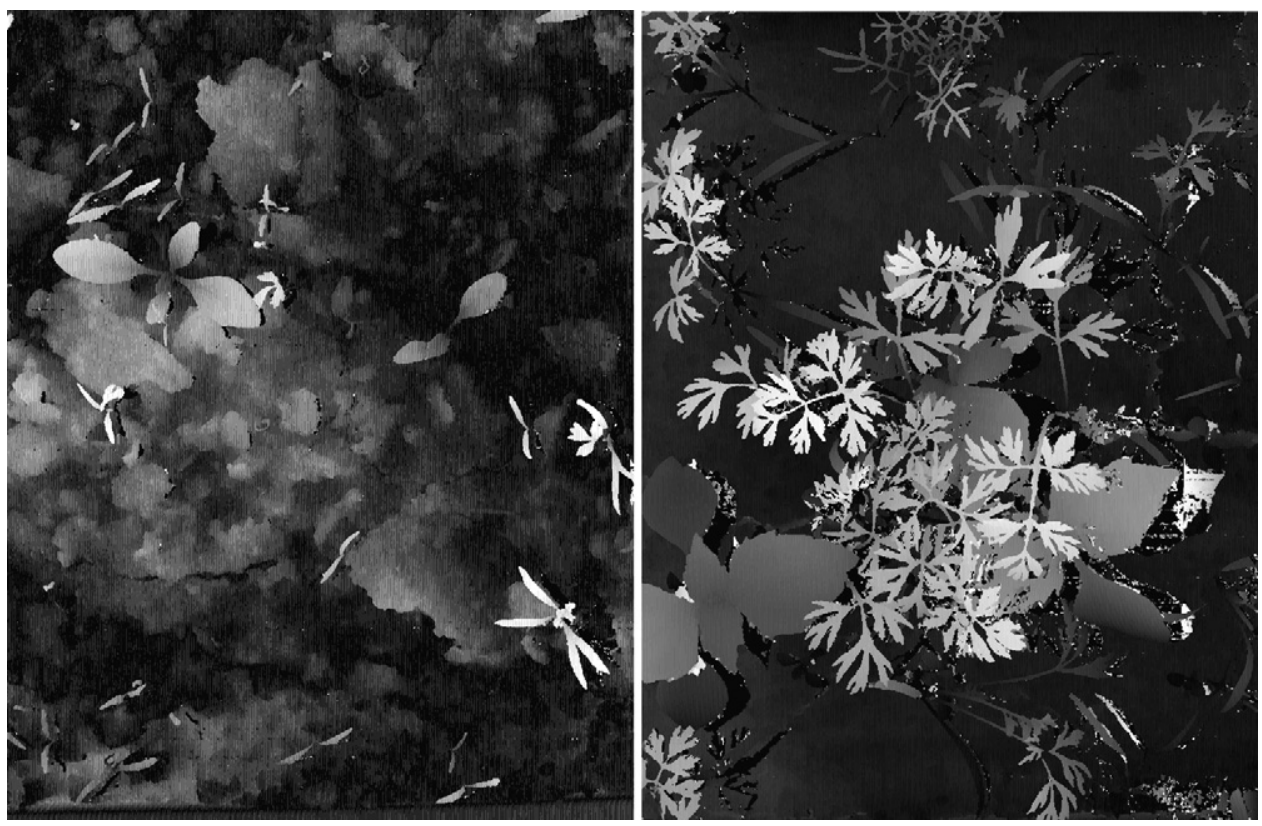

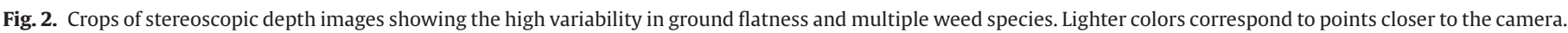

was done to give a good representation of various weed species presents as weeds tend to grow in patches and various species can be present at different times and spots over the measurement period. The number of images acquired per day varied according to meteorological conditions: strong winds made the acquisition of images difficult because of the movement of plants and/or acquisition device.

\subsection{Combination of multispectral and stereoscopic information}

In Piron et al. (2008a), it was shown that the best combination of filters for detecting various weed species located within carrot rows were respectively centered on 450,550 and $700 \mathrm{~nm}$.

Furthermore, the study of the plants stereoscopic data allowed classifying plants according to their height $(h)$ and the number of days after sowing (ND). The height parameter took into account the distance between plant pixels and the actual ground level and was insensitive to camera placement (Piron et al., 2008b).

For the combined use of multispectral and stereoscopic information, two approaches were tested, both based on quadratic discriminant analysis (QDA). In both of them, five features were selected, namely three spectral bands data, $h$ and ND. The difference between the two approaches concerned the spectral bands used. In the first approach (A), the spectral bands were centered on 450,550 and $700 \mathrm{~nm}$ (fA1, fA2, fA3, respectively) as determined by Piron et al. (2008a), independently from $h$ and ND. In the second approach (B), the three spectral bands (fB1, fB2, fB3) were selected from all the available bands (22 spectral bands between 400 and $1000 \mathrm{~nm}$, see Table 2) taking simultaneously into account $h$ and ND. The selection process consisted in evaluating the efficiency of all possible combinations of three filters, $h$ and ND.

In each approach, manually segmented data were used as a training set for the classifier, and resubstitution validation was used. The manual segmentation between crop and weeds was carried out in three steps: first, ground-plants segmentation resulting in a clean plant mask for each multispectral image, second, segmentation between crop and weeds by manually creating crude masks over the multispectral images over each type of plant, in a computer drawing program and third, creation of clean plant masks for each class (crop and weeds) by a logical AND operation on the corresponding masks from the first and second steps.

Classification accuracy (CA) represents the pixels correctly classified either as carrots or weeds, expressed as a percentage of the true carrots or weeds pixels. Overall classification accuracy, which is the mean value of both CA, was the criteria to assess feature combination efficiency.

\subsection{Sown band detection}

Detection of the sown band was performed for two purposes. First, it was used to reduce the area of the field where high precision weeding would be necessary (in the area outside the sown band, all plants can be destroyed without further characterization). Second, it was found useful in the automatic determination of crop height.

Just after sowing, the sown band is centered on the ridge because of the sowing apparatus construction. However, erosion from wind

Table 2

Filters used in the multispectral device.

\begin{tabular}{lc}
\hline Centre $(\mathrm{nm})$ & FWHM $(\mathrm{nm})$ \\
\hline 450 & 50 \\
450 & 80 \\
500 & 40 \\
500 & 80 \\
550 & 50 \\
550 & 80 \\
600 & 50 \\
600 & 80 \\
650 & 40 \\
650 & 80 \\
700 & 50 \\
700 & 80 \\
750 & 40 \\
750 & 80 \\
800 & 50 \\
800 & 100 \\
850 & 40 \\
850 & 100 \\
900 & 40 \\
900 & 100 \\
950 & 40 \\
950 & 100 \\
\hline
\end{tabular}


Table 3

Comparison of $\mathrm{CA}(\%)$ for multispectral and stereoscopic data combination approaches A and B and for multispectral and stereoscopic approaches alone.

\begin{tabular}{|c|c|c|c|c|c|}
\hline & \multicolumn{5}{|l|}{ Feature sets } \\
\hline & $\mathrm{fA} 1, \mathrm{fA} 2, \mathrm{fA} 3$ & $\mathrm{fB} 1, \mathrm{fB} 2, \mathrm{fB} 3$ & $h, \mathrm{ND}$ & Approach A (fA1, fA2, fA3, $h, \mathrm{ND}$ ) & Approach B (fB1, fB2, fB3, $h, \mathrm{ND})$ \\
\hline CA overall & 72 & 71 & 82 & 84 & 84 \\
\hline CA weeds & 78 & 80 & 80 & 81 & 83 \\
\hline CA carrots & 66 & 62 & 85 & 88 & 86 \\
\hline
\end{tabular}

or rain, machine passage can modify that central position. It was thus necessary to determine more precisely the position of the sown band at any growth stage.

Several existing crop row determination methods rely on the summation of plant pixels along an image axis to form a signal. The centers of the crop rows are supposed to be the local maxima of the generated signal (Perez et al., 2000; Sogaard and Olsen, 2003; Jones et al., 2008). Those techniques however assume that the signal has strong peaks or that a function with local peaks (e.g. a sinusoidal signal) can be fitted reasonably well to the signal. In our case, because of the presence of weeds and different growth stages, the sown band generated a noisy rectangular signal of unknown width where the local maxima were irrelevant. It was thus necessary to use a different approach in the signal analysis.

The signal used for detecting the sown band was defined by:

$S(j)=\sum_{i=1}^{1280} X(i, j) \cdot p(i, j)$

where $X(i, j)$ were the pixels of binary image for which $i$ and $j$ are the line and column respectively and $X=1$ for plant pixels and 0 otherwise. An image dilation operation was performed to limit the influence of large weeds that have a large foliage surface. The general orientation of the sown band was along the column direction of the image and $p(i, j)$ was a weight function based on the multispectral classification results. $p(i, j)$ could take two values depending on the assigned class. If the pixel was classified as crop, the value was taken as 0.66 (corresponding to crop CA in multispectral classification, see Table 3 ), if the pixel was classified as weed, the value was 0.22 (corresponding to weed classification error in multispectral classification). For each image, the limits of the sown band along the column coordinates were determined by computing the crosscorrelation between $S(j)$ and two square step signals (one up and one down) for detecting the beginning and the end of the sown band, respectively. This approach is based on the assumption that the signal generated by the carrots, which are uniformly sown, would theoretically be a single square pulse signal if weeds were not presents. Weeds are thus considered as a noise source in the generated signal.

\subsection{Probability density function estimation and peak detection}

The probability density functions (PDF) were estimated by using the ksdensity function based on a normal kernel function (Matlab, Mathworks). The window width parameter of the kernel used by the method is by default a function of the number of measurements.

For peak detection and width determination in the probability density functions, first, the global maximum was found and assumed to correspond to crop. Then, the width of the peak was defined by global maximum and minimum in the derivative of the probability density relative to height. If the limits of the peak were not around the global extrema, the next possible limit or limits were computed (second global extremum or extrema).

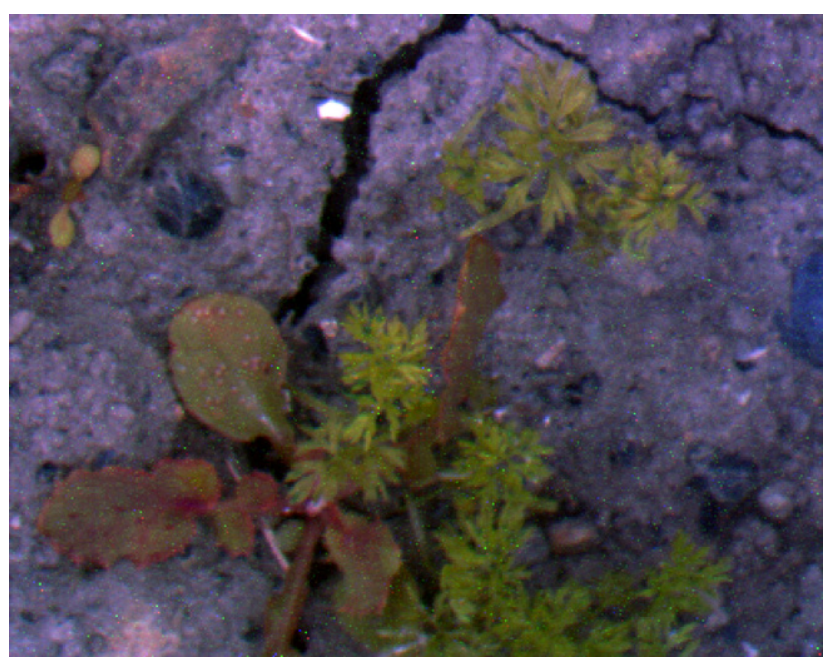

Fig. 3. Multispectral image in simulated colors showing a creeping weed under carrot plants.

\section{Results and discussions}

\subsection{Combination of multispectral and stereoscopic data}

The classification results of both approaches are summarized in Table 3. The $\mathrm{CA}^{1}$ obtained with both combination approaches was better than that obtained with multispectral approach alone and was slightly improved when compared to plant height method alone. Combination approaches $\mathrm{A}$ and $\mathrm{B}$ improved the classification of cotyledons that were nearly systematically misclassified with the purely multispectral approach but correctly classified in the stereoscopic approach.

The ability of approaches A and B to obtain a high CA was similar, even if the CA of carrots was higher with A while the CA of weeds was better with $B$. In approach $B$, the features set spectral bands ( $\mathrm{fB} 1, \mathrm{fB} 2, \mathrm{fB} 3$ ) are centered on the same wavelengths as the spectral bands from the feature set of approach $A$ (fA1, fA2, fA3) but are of different widths. This may be explained by possible correlation between color and weeds height. Indeed, creeping weeds that were correctly classified by both methods represented a large part of the data set due to their size and number (Fig. 3).

\subsection{Analysis of stereoscopic plant data over the entire ridge width}

For each measurement day, the PDF of plants including crop and weeds heights were built. The PDF of crop and weeds considered separately resulted from manually segmented data obtained on the whole ridge width. During the growth, an evolution in the PDF of the plants was clearly apparent (Fig. 4).

\footnotetext{
1 The CA is computed over the whole considered measurement period, it not mean of single CA for each image or measurement day. Reported CA are always for the optimal combination of features returned by the feature selection method.
} 

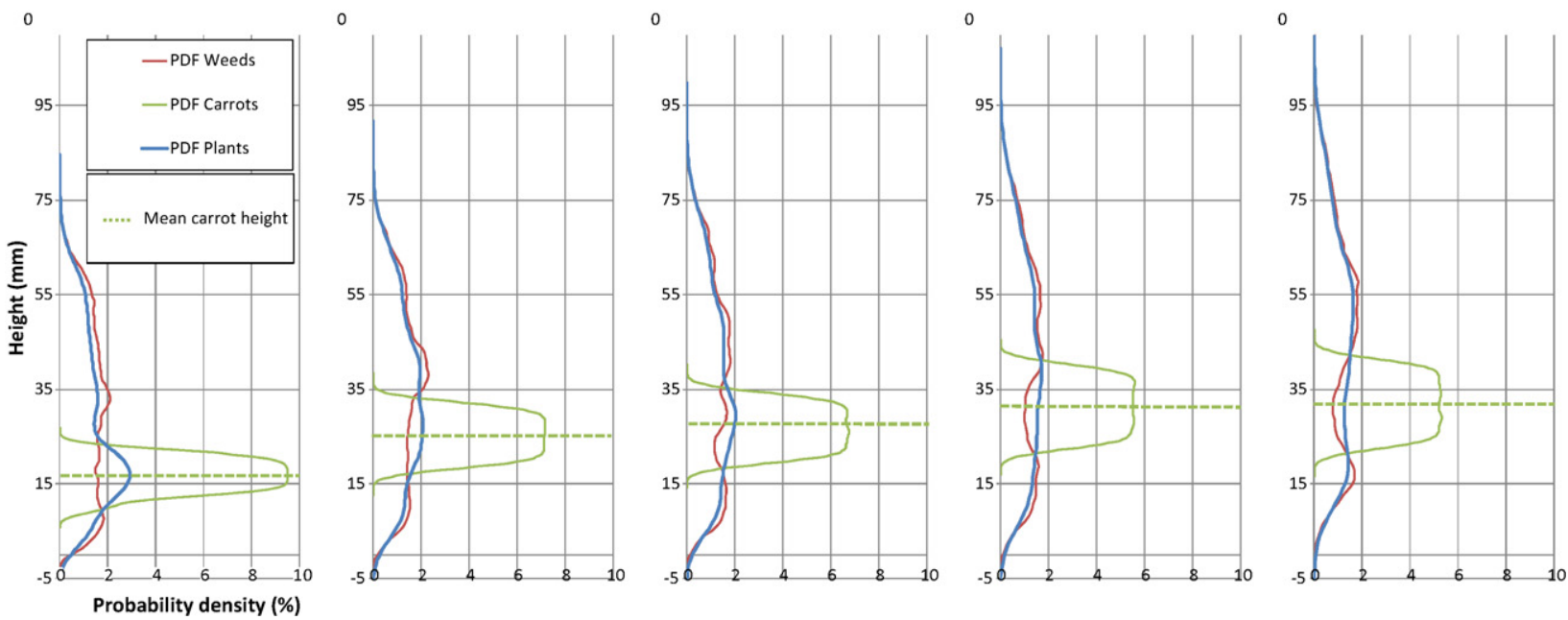

Fig. 4. PDF of weeds, carrots and plants, over the whole ridge at, from left to right, 21, 28, 3037 and 39 days after sowing.

\subsubsection{Crop height distribution}

The probability density functions of the carrots were not very widespread. During the measurement period, the average carrot height grew from 17 to $32 \mathrm{~mm}$. The shape of the distribution changed with growth and tended to become more flat, which corresponded to the plants occupying a larger height range (Table 4).

\subsubsection{Weeds height distribution}

The PDF of weeds were not as regularly shaped or narrow as the crop ones, because the weed class was a mixture including different species of various sizes and growths. The PDF showed a small first peak for low heights corresponding likely to creeping weeds. Another peak was also noticeable for larger weed heights. Both peaks were moving towards greater heights as growth progressed.

\subsubsection{Probability density functions analysis for weed detection}

The potential for weed detection on height basis can be deduced from the crop and weed PDF. The optimal method consists in classifying plant pixels according to their height higher probability. The optimal limits for differentiating crop from weed were the points with equal probabilities for a plant of being either a carrot or a weed (Table 5). Given the shape of the crop and weeds PDF, this defined a height range in which the plants were more likely to be carrots than weeds. Outside this range the plants are more likely to be weeds.

Table 6 gives a comparison between the optimal and automatic methods in terms of classification accuracies (CA). The classification accuracy for a class is the percentage of observations correctly classified. The overall CA is the mean of the CA of the weed and carrot classes.

Because the range for weed detection varied with growth and was unlikely to be constant between fields, seasons or even in a field, it was necessary to determine the range limits automatically.

However, while this was evident when looking at the PDF for crop and weeds, those were the product of manual segmentation. The information available to make this task autonomous (free of human input) was the plant PDF which contained less information.

Table 4

Descriptive statistics for carrot height distribution over the whole ridge ( $\mathrm{mm}$ ).

\begin{tabular}{lccccc}
\hline Days after sowing & 21 & 28 & 30 & 37 & 39 \\
\hline Average height & 17 & 25.5 & 27 & 31.5 & 32 \\
Standard deviation & 3.25 & 4 & 4.5 & 5.2 & 5.5 \\
Interquartile range & 5 & 7.5 & 7.5 & 9 & 9.5 \\
\hline
\end{tabular}

Table 5

Comparison of parameters for the height base detection and probabilities of each class being in the height range ( $\mathrm{mm}$ and \%, respectively).

\begin{tabular}{lcclll}
\hline Days after sowing & 21 & 28 & 30 & 37 & 39 \\
\hline Automatic method & & & & & \\
$\quad$ Width & 17.5 & 20 & 22 & 11.5 & 22 \\
Center & 18.25 & 25 & 27 & 39.25 & 58 \\
Lower limit & 9.5 & 15 & 16 & 33.5 & 47 \\
Upper limit & 27 & 35 & 38 & 45 & 69 \\
Pr weeds & 16 & 30 & 26 & 18 & 34 \\
Pr carrots & 84 & 90 & 89 & 36 & 0 \\
Optimal method & & & & & \\
Width & 14 & 16 & 16 & 21 & 21.5 \\
Center & 16.5 & 25 & 27 & 30.5 & 31.75 \\
Lower limit & 9.5 & 17 & 19 & 20 & 21 \\
Upper limit & 23.5 & 33 & 35 & 41 & 42.5 \\
Pr weeds & 20 & 26 & 25 & 28 & 23 \\
Pr carrots & 96 & 96 & 89 & 96 & 97 \\
\hline
\end{tabular}

There first appeared to be insufficient information to detect a peak corresponding to carrots except for the first measurement day.

The plant PDF can be seen as a mixture of the crop and weeds PDF. To have the carrot heights appear more readily in the plant PDF, they had to be more represented. To achieve this, we chose to restrict the PDF building process to the sown band where the proportion of carrot plants to weeds was more favorable.

\subsection{Analysis of stereoscopic plant data in the sown band}

\subsubsection{Sown band detection}

Fig. 5 shows detected sown bands. The sown band detection algorithm did not perform as well at very early growth stages, when

Table 6

Comparison of $\mathrm{CA}(\%)$ for height based detection, per measurement day.

\begin{tabular}{lllllr}
\hline Day & 21 & 28 & 30 & 37 & 39 \\
\hline Automatic method & & & & & \\
CA overall & 84 & 81 & 82 & 61 & 20 \\
CA weeds & 84 & 88 & 87 & 56 & 40 \\
CA carrots & 84 & 75 & 77 & 67 & 0 \\
Optimal method & & & & & \\
CA overall & 89 & 87 & 83 & 86 & 89 \\
CA weeds & 95 & 95 & 87 & 95 & 96 \\
CA carrots & 83 & 79 & 78 & 77 & 81 \\
\hline
\end{tabular}




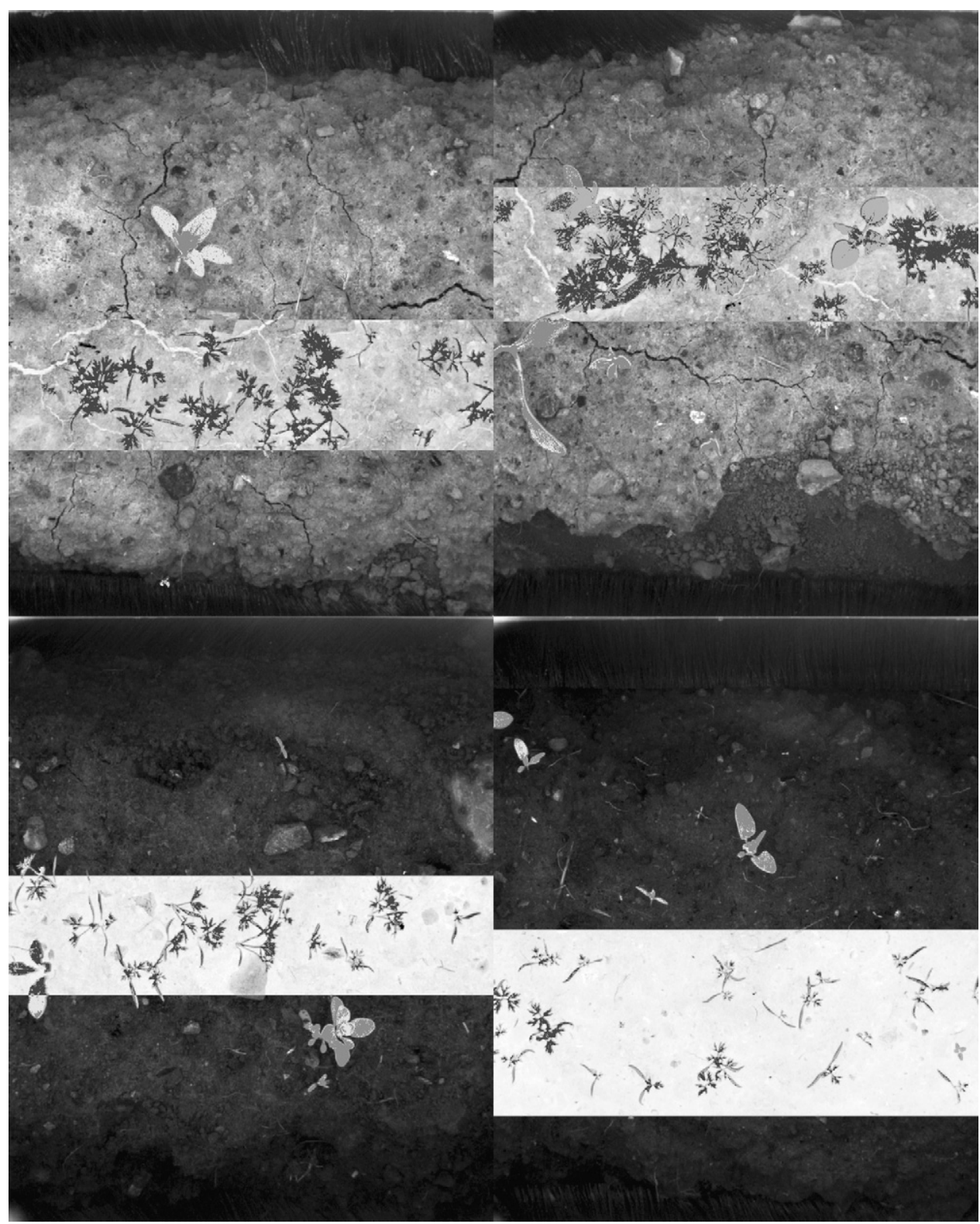

Fig. 5. Examples of sown band detection.

the crop comprised mostly cotyledons. However, this had minor influence on classification results since the peak in the PDF corresponding to crop is already visible at that stage before sown band detection (Fig. 4).

Because of the relative sizes of the ridge and sown band, it was estimated that approximately $75 \%$ of the weeds were located outside of the sown band. This number probably decreased as erosion caused by weather or machine reduced the width of the ridge. The plant, crop and weeds PDF were then built with the height source data restricted to the detected sown band.

\subsubsection{Crop and weeds distribution}

Unsurprisingly, the crop and weeds PDF were very similar in shape to the ones found over the whole ridge (Fig. 6). The carrots PDF were nearly identical because nearly all carrots were in the sown band and weed species were spatially randomly distributed. The plant PDF restricted to the sown band however exhibited more visible peaks corresponding to the crop until 30 days after sowing compared to the plant PDF over the whole ridge.

\subsubsection{PDF Analysis for weed detection}

Using the peak detection method described in Section 2.4, a height range was determined for each measurement day. The height ranges resulting from this method were compared with the optimal method (Table 5). Also given in the same table were the probabilities of having crop and weed in the height ranges for each method, per measurement day. Table 6 gives the CA per measurement day.

The comparison between the optimal and automatic methods for crop height detection showed that the automatic method was sucessfull for early stages (21, 28 and 30 days) but not for later growth stages ( 37 and 39 days after sowing). The lack of a visible peak in the probability density, even when using the sown band detection method, was caused by the diminishing relative surface of crop compared to weed. It should be noted however that in current practice, fields are usually weeded before weeds attain such sizes, as a late weeding can negatively effect yield.

Tables 7 and 8 give the CA for the three methods for the same sample and period as in Piron et al. (2008b). Previous classification results relied on QDA. To allow comparison with the previously 

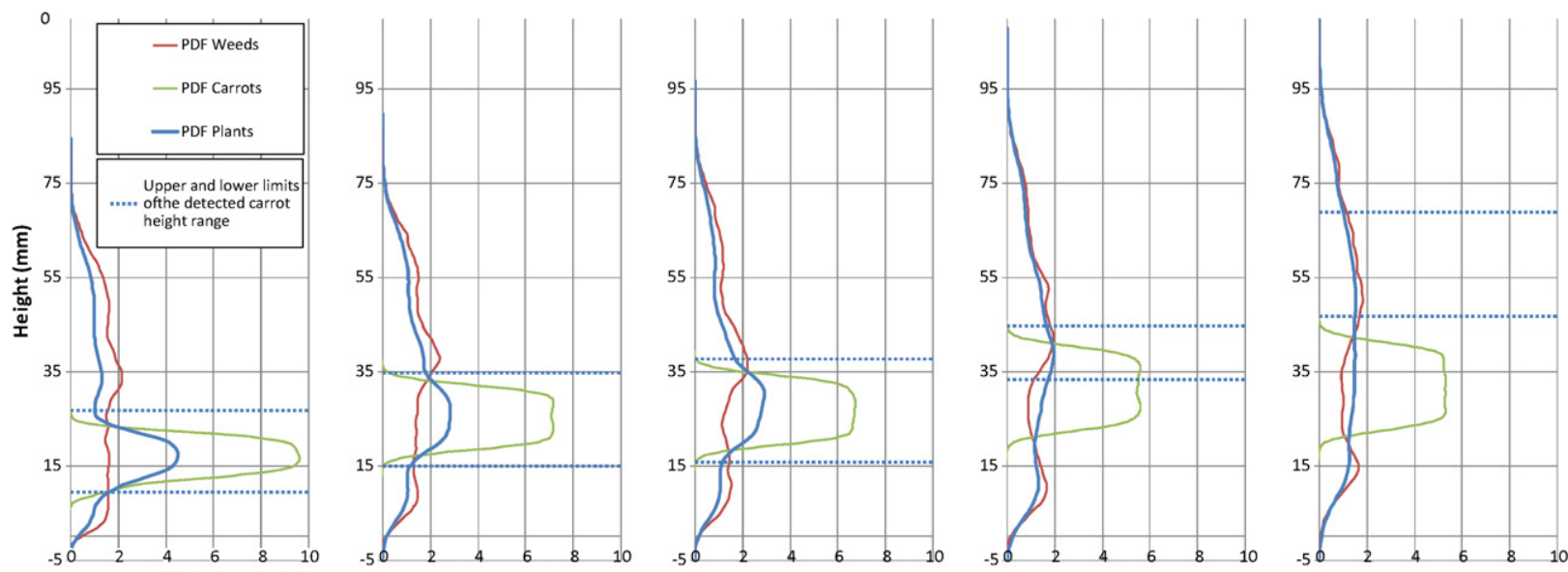

Fig. 6. PDF of weeds, carrots and plants, restricted to sown band at, from left to right, 21, 28, 3037 and 39 days after sowing.

Table 7

Comparison of $\mathrm{CA}(\%)$ for height based detection over the whole measurement period.

\begin{tabular}{llll}
\hline & $\begin{array}{l}\text { QDA } \\
\text { classification }\end{array}$ & $\begin{array}{l}\text { Automatic } \\
\text { classification }\end{array}$ & $\begin{array}{l}\text { Optimal } \\
\text { classification }\end{array}$ \\
\hline CA overall & 82 & 66 & 86 \\
CA weeds & 80 & 71 & 93 \\
CA carrots & 85 & 61 & 79 \\
\hline
\end{tabular}

Table 8

Comparison of $\mathrm{CA}(\%)$ for height based detection for measurements until 30 days after sowing.

\begin{tabular}{llll}
\hline & $\begin{array}{l}\text { QDA } \\
\text { classification }\end{array}$ & $\begin{array}{l}\text { Automatic } \\
\text { classification }\end{array}$ & $\begin{array}{l}\text { Optimal } \\
\text { classification }\end{array}$ \\
\hline CA overall & 80 & 82 & 86 \\
CA weeds & 79 & 86 & 92 \\
CA carrots & 83 & 78 & 80 \\
\hline
\end{tabular}

studied method, the CA are given over the whole measurement period which explains the bad results for the automatic method since it has been established that this method did not work for later growth stages. When the CA evaluation was restricted to the measurement period for which the automatic classification method proved to be efficient, the comparison was more meaningful. The automatic classification method gave results only slightly inferior to the optimal classification as defined earlier.

\section{Conclusion}

Multispectral and stereoscopic methods have both potential to detect weeds from carrots. In this study, in a first stage, the combination of multispectral and height features for weed detection was examined using two approaches. In approach A, the best combination of filters selected independently from any other feature was used (filters centered at 450, 550 and $700 \mathrm{~nm}$ ), and was completed with two other parameters: plant height and number of days after sowing. In approach $B$ the best combination of filters taking into account plant height and number of days after sowing during the selection process was computed and used conjointly with plant height and number of days after sowing. An improvement over either multispectral or height features and number of days after sowing taken separately was found but was however limited. The more relevant feature to classify weeds from carrots was clearly the plant height and number of days after sowing.

In a second stage, from the stereoscopic data analysis, it was found that determination of height limits between crop and weeds could become independent from statistical training or operator input. The proposed method was based on the construction of the probability density function of plant height from stereoscopic multispectral images limited to the sown band. This approach gave results close to optimal classification for measurements up to 30 days after sowing (CA of 82 and $86 \%$, respectively).

\section{Acknowledgement}

This project is funded by the "Ministère de la Région wallonne, DGTRE”, project “RECADVEN" no. EPH3310300R049F/41J81.

\section{References}

Andersen, H.J., Reng, L., Kirk, K., 2005. Geometric plant properties by relaxed stereo vision using simulated annealing. Computers and Electronics in Agriculture 49 (2), 219-232.

Borregaard, T., Nielsen, H., Norgaard, L., Have, H., 2002. Crop-weed discrimination by line imaging spectroscopy. Journal of Agricultural Engineering Research 75, 389-400.

Feyaerts, F., van Gool, L., 2001. Multi-spectral vision system for weed detection. Pattern Recognition Letters 22, 667-674.

Franz, E., Gebhardt, M.R., Unklesbay, K., 1991. The use of local spectral properties of leaves as an aid for identifying weed seedlings in digital images. Transactions of the ASAE 34 (2), 682-687.

He, D.X., Matsuura, Y., Kozai, T., Ting, K.C., 2003. A binocular stereovision system for transplant growth variable analysis. Applied Engineering in Agriculture 19 (5), 611-617.

Jones, G., Gée, C., Truchetet, F., 2008. Modelling agronomic images for weed detection and comparison of crop/weed discrimination algorithm performance. Precision Agriculture, http://dx.doi.org/10.1007/s11119-008-9086-9.

Nielsen, M., Andersen, H., Slaughter, D., Giles, D., 2004. Detecting leaf features for automatic weed control using trinocular stereo vision. In: International Conference on Precision Agriculture 2004, Minneapolis, MN, USA.

Perez, A., Lopez, F., Benlloch, J.V., Christensen, S., 2000. Colour and shape analysis techniques for weed detection in cereal fields. Computers and Electronics in Agriculture 25, 197-212.

Piron, A., Leemans, V., Kleynen, O., Lebeau, F., Destain, M.-F., 2008a. Selection of the most efficient wavelength bands for discriminating weeds from crop. Computers and Electronics in Agriculture 62, 141-148.

Piron, A., Leemans, V., Kleynen, O., Destain, M.-F., 2008b. Determination of plant height for weed detection in stereoscopic images. In: Agricultural Engineering Conference 2008, Hersonissos, Greece.

Sanchez, A., Marchant, J., 2000. Fusing 3D information for crop/weeds classification. In: Proceedings of the 15th International Conference on Pattern Recognition Barcelona. IEEE Computer Society Press, Spain.

Sogaard, H.T., Olsen, H.J., 2003. Determination of crop rows by image analysis without segmentation. Computers and Electronics in Agriculture 38 (2), 141-158.

Vrindts, E., De Baerdemaeker, J., Ramon, H., 2002. Weed detection using canopy reflection. Precision Agriculture 3, 63-80.

Wang, N., Zhang, N., Dowell, F.E., Sun, Y., Peterson, D.E., 2001. Design of an optical weed sensor using plant spectral characteristics. Transactions of the ASAE 44 (2), 409-419.

Zhang, N., Chaisattapagon, C., 1995. Effective criteria for weed identification in wheat fields using machine vision. Transactions of the ASAE 38, 965-974. 